\title{
Patterns and outcomes of immune-related adverse events in solid tumor patients treated with immune checkpoint inhibitors in Thailand: a multicenter analysis
}

\author{
Nuttapong Ngamphaiboon ${ }^{1}$, Suthinee Ithimakin ${ }^{2}$, Teerada Siripoon ${ }^{1}$, Nattaya Sintawichai ${ }^{3}$ and
} Virote Sriuranpong ${ }^{3^{*}}$

Presentation: The abstract of this study was partially presented at the Korean Society of Clinical Oncology 2019.

\begin{abstract}
Background: Most immune-related adverse event (irAE) patterns and treatment guidelines are based on western clinical data. We evaluated the incidence and patterns of irAEs in patients treated with immune-checkpoint inhibitors (ICl) in Thailand.

Methods: All solid tumor patients treated with ICls were retrospectively reviewed in a multicenter analysis. The study aims to evaluate the incidence of irAEs and their characteristics, treatments, outcomes, and impact on survival. All irAEs were graded using the CTCAE version 4.0. Characteristics of irAEs including time to onset, duration of irAEs, specific treatments, and outcomes of irAEs were reviewed. The Chi-square or Fisher's exact test was used to compare variables. Overall survival (OS) was estimated by the Kaplan-Meier method, and compared by the log-rank test. A $p$-value $<0.05$ was considered statistically significant.

Results: irAEs of any grade were observed in 98 of 414 patients (24\%), whereas grades 3-4 irAEs were observed in $5.6 \%$. The majority of patients (78\%) were treated with monotherapy ICI (anti-PD1/PD-L1 92\%). The most common all-grade irAEs were hypothyroidism (7.5\%), hepatitis (6.5\%), and rash (4.8\%). Median onset of overall irAEs was 63 days. Pancreatitis and pneumonitis had the earliest onset at 30 and 34 days, respectively. ICIs were rechallenged in 68 of 98 patients with irAE. Eleven of sixty-eight patients (11.2\%) with initial irAE had reoccurrence after ICI rechallenge. Based on a multivariate analysis, pre-existing hypothyroidism, ICl used in a clinical trial setting, and combinations of $\mathrm{ICl} / \mathrm{ICl}$ were independent factors predicting irAE occurrence. Patients with irAE had a statistically significant longer overall survival (OS) when compared to patients without irAE $(p=0.019)$. A multivariate analysis revealed that occurrence of irAE was an independent prognostic factor for OS (HR 0.70,95\% Cl 0.51-0.96; $p=0.028)$.
\end{abstract}

\footnotetext{
*Correspondence: virote.s@chula.ac.th

${ }^{3}$ Division of Medical Oncology, Department of Internal Medicine, Faculty of Medicine, Chulalongkorn University \& The King Chulalongkorn

Memorial Hospital, 1873 Rama IV Rd., Pathumwan, Bangkok 10330,

Thailand

Full list of author information is available at the end of the article
}

(C) The Author(s) 2021. Open Access This article is licensed under a Creative Commons Attribution 4.0 International License, which permits use, sharing, adaptation, distribution and reproduction in any medium or format, as long as you give appropriate credit to the original author(s) and the source, provide a link to the Creative Commons licence, and indicate if changes were made. The images or other third party material in this article are included in the article's Creative Commons licence, unless indicated otherwise in a credit line to the material. If material is not included in the article's Creative Commons licence and your intended use is not permitted by statutory regulation or exceeds the permitted use, you will need to obtain permission directly from the copyright holder. To view a copy of this licence, visit http://creativecommons.org/licenses/by/4.0/. The Creative Commons Public Domain Dedication waiver (http://creativeco mmons.org/publicdomain/zero/1.0/) applies to the data made available in this article, unless otherwise stated in a credit line to the data. 
Conclusion: irAE was commonly observed in Thai cancer patients treated with ICls. Most irAEs were low-grade and manageable. Re-occurrence of irAE after re-challenging ICI was not uncommonly observed. Patients who experienced irAEs might have significantly longer OS compared to patients without irAEs. However, OS in this study should be interpreted with caution since it might be affected by various tumor types, treatment settings, dosing schedule, and $\mathrm{ICl}$ combinations.

Keywords: cancer immunotherapy, Immune-checkpoint inhibitors, Immune-related adverse events, Overall survival, Thailand

\section{Background}

Cancer immunotherapy, specifically immune checkpoint inhibitors (ICI), are used in monotherapy, combination therapy, or jointly with chemotherapeutic agents and/ or targeted therapies. They have become the standard of care for various types of cancers due to significant improvement of clinical and long-term survival outcomes [1].

Use of ICIs can lead to a broad spectrum of autoimmunity-like symptoms - termed immune-related adverse events (irAEs) - that can affect multiple organs, most commonly the endocrine glands, gastrointestinal tract, liver, and skin [2]. Thus, irAEs present a significant challenge in clinical practice, yet the mechanisms underlying irAE development remain unclear. Several potential mechanisms include (i) increasing T-cell activity against common antigens present in both tumors and normal tissue, (ii) increasing levels of pre-existing autoantibodies, (iii) increasing the levels of proinflammatory cytokines, and (iv) enhancing complement-mediated inflammation due to direct binding of an anti-CTLA-4 antibody with CTLA-4 expressed on normal tissue, such as the pituitary gland [2].

The severity of irAEs range from asymptomatic, to mild, to life-threatening [3]. Characteristics and patterns of irAEs may also vary, depending on the ICI's mechanism of action and the combination used [3]. Moreover, factors predictive of irAEs in ICI-treated cancer patients remain largely unknown [2], though several have been proposed, such as germline genetic factors and host microbiota composition [4-6]. Though variation in incidence and patterns of irAEs in different ethnicities have not been well studied, a sub-group analysis of Asian vs. non-Asian populations in several prospective Phase III studies reported different incidences of specific irAEs between these two populations [7-11]. For instance, a Phase III KeyNote-048 study of pembrolizumab with or without chemotherapy in patients with recurrent/metastatic head and neck squamous cell carcinoma reported that immune-related pneumonitis was more prominent in non-Asians when compared with the Asian population [7]. Moreover, a Phase III CheckMate-025 study of nivolumab monotherapy in advanced renal cell carcinoma reported that the incidence of any-grade endocrine and renal related irAEs was lower, whereas hepatic-related irAE incidence was higher in Japanese subjects when compared to the global population [9]. To date, there is no data of irAE characteristics and outcomes reported in Thai cancer patients treated with ICIs. Therefore, in a large multicenter study, we evaluated the incidence and clinical patterns of irAEs in ICI-treated cancer patients in Thailand to explore factors predictive of immune-related toxicity in high-risk patients, and outcomes of irAE treatment and survival.

\section{Methods}

\section{Study design}

All patients with solid tumors who were treated with any immune checkpoint inhibitors at 3 cancer centers: King Chulalongkorn Memorial, Siriraj, and Ramathibodi hospitals between November 2013 to December 2019 were identified through each center's database. Available medical records were then retrospectively reviewed using a central database and standardized electronic case record forms. Hematologic malignancy and patients treated with cell or therapeutic vaccine therapy were excluded. Patient baseline characteristics including age and sex, smoking status, Eastern Cooperative Oncology Group (ECOG) performance status, comorbidities of interest, and tumor types were abstracted. Tumor histology, PD-L1 expression, and previous treatments were reviewed. For patients who tested for PD-L1 expression, PD-L1 assay and cut-off for positivity was defined based on standard criteria for each anti-PD1/PD-L1 drugs. Survival outcomes were also collected. Overall survival (OS) was defined as the duration from date of immunecheckpoint inhibitor treatment initiation to death or last follow-up. The survival status of patients was verified and crosschecked with the National Security Death Index. Ethics approval was obtained through either the ethics committee (EC) at each study center. All procedures performed in this study were in accordance with the ethical standards of the institutional research committee and with the 1964 Helsinki declaration and its later amendments or comparable ethical standards. 


\section{Immunotherapy treatment}

Eligible patients treated with any immune checkpoint inhibitors in clinical trials, compassionate usage, and clinical practice settings were included in the study. Immune checkpoints inhibitors evaluated in this study included anti-PD1, anti-PD-L1, and anti-CTLA-4. The dose and schedule of each immune checkpoint inhibitor in this study were determined by recommended dose per clinical trial or standard recommended dose in compassionate usage programs or clinical practice. However, for patients who received immune checkpoint inhibitors in a clinical practice setting, the dose and schedule of each drug might be adjusted at the treating physician's discretion.

\section{Immune related adverse events}

All treatment related toxicities, including irAEs, were graded using the Common Terminology Criteria for Adverse Events (CTCAE) version 4.0 [12]. All irAEs were categorized based on primary organ involvement and maximum grade by CTCAE v.4.0. Characteristics of irAEs including time to onset, duration of irAEs, specific treatments, and outcomes of irAEs were reviewed. irAEs were considered fully resolved when the irAEs return to grade 0 without any treatment-specific medications such as systemic steroids, thyroxine supplements, or anti-thyroid drugs. irAEs were managed and treated according specific guidelines for patients in clinical trials, whereas patients with irAEs outside of clinical trials were treated following standard recommendations and guidelines available at the time of irAE diagnosis at the treating physician's discretion [13-16].

\section{Statistical analysis}

The study aims to evaluate the incidence of irAEs and their characteristics, treatments, and outcomes in Thai patients who received ICIs as a primary objective. An impact of irAE occurrence on survival was evaluated as a secondary objective. Baseline demographic data was described using descriptive analysis. Categorical variables were presented as number of patients and percentage then Chi-square or Fisher's exact test was used to compare variables between the irAE and no irAE groups.

Overall survival (OS) was estimated by the KaplanMeier method, and the log-rank test was used to compare between irAE and no irAE groups. Univariate Cox regression analysis was performed to identify prognostic factors for OS. Factors with $p$-values $<0.1$ from the univariate Cox regression analysis along with irAE, setting of treatment, and ICI use were considered in a multivariate analysis.
To identify factors associated with ir AE occurrence, simple logistic regression analysis was used. Then factors with $p$-values less than 0.1 from the simple logistic regression analysis were considered in a multiple logistic regression analysis. All analyses were done on STATA version 15 (Stata Corp., College Station, Texas, USA). A $p$-value $<0.05$ was considered statistically significant.

\section{Results \\ Baseline characteristics}

A total of 414 eligible patients were identified from the 3 centers. Baseline patient characteristics are listed in Table 1. Overall, irAEs of any grade occurred in 98 of 414 patients $(23.7 \%)$. Elderly patients (age $\geq 65$ yearsold) $(54.6 \%)$, and males $(69.3 \%)$ made up the majority of ICI-treated individuals in this study. Primary lung cancer (46.6\%) and adenocarcinoma (51.2\%) were the most common tumor types in the study. Among patients who were tested for PD-L1 expression, 70 of 109 patients tested positive (64\%), although the majority of patients in the study were not tested for PD-L1 expression. 167 of 414 patients received prior radiotherapy before ICI treatment was initiated. Only 32 and 15 patients received previous radiation to the lungs and mediastinal areas, respectively, prior to immunotherapy treatment. The majority of patients received prior (neo)adjuvant chemotherapy before initiation of ICI treatment in metastatic setting. irAEs were significantly associated with a prior history of hypothyroidism $(p<0.001)$. Majority of patients $(98.4 \%)$ had stage 4 disease. There was no statistically significant difference in smoking status, PD-L1 expression, and previous treatment among patients with and without irAEs.

\section{Immunotherapy treatment}

Most ICIs in this study (51.7\%) were delivered in a firstline palliative setting (Table 2). Only 6 patients $(1.4 \%)$ received adjuvant ICIs, and all of them were treated in a clinical trial setting. In patients who received monotherapy ICI, 25 of 322 patients (7.8\%) were treated with antiCTLA4, whereas 297 patients (92.2\%) received anti-PD1/ PD-L1. Patients who received ICIs in clinical trial settings had significantly more frequent irAEs $(60.2 \%)$ than those in compassionate use programs (5.1\%) and those in clinical practice $(34.7 \%) ; p<0.001$. Nineteen patients received combined immunotherapy including anti-PD-1/PD-L1 and anti-CTLA4. Eleven of these 19 patients (57.9\%) developed irAEs, whereas 72 of 322 patients (22.4\%) treated with single agent immunotherapy had irAEs $(p=0.004)$. 
Table 1 Patient characteristics

\begin{tabular}{|c|c|c|c|c|}
\hline Patient characteristics & All $N=414$ & No irAE $N=316$ & $\operatorname{irAE} N=98$ & $p$-value \\
\hline Median Age (range) & $63(17-97)$ & $64(17-97)$ & $63(20-91)$ & \\
\hline$<65$ years-old & $226(54.6)$ & $170(53.8)$ & $56(57.1)$ & 0.561 \\
\hline$\geq 65$ years-old & $188(45.4)$ & $146(46.2)$ & $42(82.9)$ & \\
\hline Gender & & & & 0.627 \\
\hline Male & $287(69.3)$ & $221(69.9)$ & $66(67.4)$ & \\
\hline Female & $127(30.7)$ & $95(30.1)$ & $32(32.6)$ & \\
\hline Smoking status & & & & 0.989 \\
\hline Never & $142(34.3)$ & $108(34.2)$ & $34(34.7)$ & \\
\hline Active or ex-smoker & $142(34.3)$ & $109(34.5)$ & $33(33.7)$ & \\
\hline Unknown & $130(31.4)$ & $99(31.3)$ & $31(31.7)$ & \\
\hline ECOG status & & & & 0.431 \\
\hline $0-1$ & $394(95.2)$ & $299(94.6)$ & $95(96.9)$ & \\
\hline$\geq 2$ & $20(4.8)$ & $17(5.4)$ & $3(3.1)$ & \\
\hline \multicolumn{5}{|l|}{ Comorbidity of Interest } \\
\hline Hypothyroidism & $15(3.6)$ & $6(1.9)$ & $9(9.2)$ & 0.001 \\
\hline Hyperthyroidism & $5(1.2)$ & $4(1.3)$ & $1(1.0)$ & 1.000 \\
\hline $\mathrm{DM}$ & $68(16.4)$ & $53(16.8)$ & $15(15.3)$ & 0.732 \\
\hline Chronic HBV & $28(6.8)$ & $24(7.6)$ & $4(4.1)$ & 0.355 \\
\hline Chronic HCV & $9(2.2)$ & $8(2.5)$ & $1(1.0)$ & 0.692 \\
\hline Primary Tumor types & & & & 0.031 \\
\hline Lung cancer & $193(46.6)$ & $156(49.4)$ & $37(37.8)$ & \\
\hline $\mathrm{HCC}^{\mathrm{a}}$ & $42(10.1)$ & $30(9.5)$ & $12(12.2)$ & \\
\hline Bladder cancer/TCC ${ }^{a}$ & $40(9.7)$ & $30(9.5)$ & $10(10.2)$ & \\
\hline Melanoma & $33(8.0)$ & $21(6.7)$ & $12(12.2)$ & \\
\hline $\mathrm{HNSCC}^{\mathrm{a}}$ & $23(5.6)$ & $19(6.0)$ & $4(4.1)$ & \\
\hline $\mathrm{RCC}^{\mathrm{a}}$ & $20(4.8)$ & $16(5.1)$ & $4(4.1)$ & \\
\hline Nasopharyngeal carcinoma & $19(4.6)$ & $12(3.8)$ & $7(7.2)$ & \\
\hline Esophageal cancer & $8(1.9)$ & $6(1.9)$ & $2(2.0)$ & \\
\hline Colorectal cancer & $7(1.7)$ & $4(1.3)$ & $3(3.1)$ & \\
\hline Breast cancer & $6(1.4)$ & $6(1.9)$ & 0 & \\
\hline Pancreatic cancer & $4(1.0)$ & $1(0.3)$ & $3(3.1)$ & \\
\hline Stomach/EGJa cancer & $3(0.7)$ & $1(0.3)$ & $2(2.0)$ & \\
\hline Cholangiocarcinoma & $2(0.5)$ & $1(0.3)$ & $1(1.0)$ & \\
\hline Others & $14(3.4)$ & $13(3.0)$ & $1(1.0)$ & \\
\hline Histology & & & & 0.122 \\
\hline Adenocarcinoma & $212(51.2)$ & $163(51.6)$ & $49(50.0)$ & \\
\hline Squamous Cell & $79(19.1)$ & $58(18.3)$ & $21(21.4)$ & \\
\hline Transitional cell carcinoma & $41(9.9)$ & $31(9.8)$ & $10(10.2)$ & \\
\hline Melanoma & $36(8.7)$ & $23(7.3)$ & $13(13.3)$ & \\
\hline Clear cell & $15(3.6)$ & $12(3.8)$ & $3(3.1)$ & \\
\hline Others & $31(7.5)$ & $29(9.2)$ & $2(2.0)$ & \\
\hline PD-L1 status & & & & 0.065 \\
\hline Negative & $39(9.4)$ & $29(9.2)$ & $10(10.2)$ & \\
\hline Positive & $70(16.9)$ & $61(19.3)$ & $9(9.2)$ & \\
\hline Unknown & $305(73.7)$ & $226(71.5)$ & $79(80.6)$ & \\
\hline \multicolumn{5}{|l|}{ Previous Treatment } \\
\hline Chemotherapy & $250(60.4)$ & $198(62.7)$ & $52(53.1)$ & 0.090 \\
\hline Radiotherapy & $167(40.3)$ & $128(40.5)$ & $39(39.8)$ & 0.900 \\
\hline \multicolumn{5}{|l|}{ Site of previous Radiotherapy } \\
\hline Lung & $32(7.7)$ & $24(7.6)$ & $8(8.2)$ & 0.854 \\
\hline Mediastinum & $15(3.6)$ & $10(3.2)$ & $5(5.1)$ & 0.370 \\
\hline Neck/thyroid & $24(5.8)$ & $17(5.4)$ & $7(7.1)$ & 0.514 \\
\hline
\end{tabular}

${ }^{a}$ TCC transitional cell carcinoma, HCC hepatocellular carcinoma, HNSCC head and neck squamous cell carcinoma, RCC renal cell carcinoma 
Table 2 Immunotherapy Treatment

\begin{tabular}{|c|c|c|c|c|}
\hline Treatment & All $N=414$ & No irAE $N=316$ & $\operatorname{irAE} N=98$ & $p$-value \\
\hline Settings & & & & $<0.001$ \\
\hline Clinical trial & $161(38.9)$ & $102(32.3)$ & $59(60.2)$ & \\
\hline Compassionate program & $31(7.5)$ & $26(8.2)$ & $5(5.1)$ & \\
\hline Clinical practice & $222(53.6)$ & $188(59.5)$ & $34(34.7)$ & \\
\hline Treatment setting & & & & 0.074 \\
\hline Adjuvant & $6(1.4)$ & $5(1.6)$ & $1(1.0)$ & \\
\hline 1st line metastasis & $214(51.7)$ & $152(48.1)$ & $62(63.3)$ & \\
\hline 2nd line metastasis & $113(27.3)$ & $92(29.1)$ & $21(21.4)$ & \\
\hline$\geq 3$ rd line metastasis & $81(19.6)$ & $67(21.2)$ & $14(14.3)$ & \\
\hline Immunotherapy combination & & & & 0.004 \\
\hline Single agent & $322(77.8)$ & $250(79.1)$ & $72(73.5)$ & \\
\hline Combination $\mathrm{ICl}+\mathrm{ICl}$ & $19(4.6)$ & $8(2.5)$ & $11(11.2)$ & \\
\hline Combination $\mathrm{ICI}+$ chemotherapy & $62(15.0)$ & $50(15.8)$ & $12(12.2)$ & \\
\hline Combination $\mathrm{ICl}+$ others & $11(2.7)$ & $8(2.5)$ & $3(3.1)$ & \\
\hline
\end{tabular}

$\mathrm{ICl}$ immune checkpoint inhibitor, ir $A E$ immune related adverse events

\section{Immune-related adverse events, their treatments and outcomes}

3The overall incidence of all grade irAEs in this study was 98 of 414 patients (23.7\%) (Table 3 ). The most frequently occurring all grade irAEs were hypothyroidism (7.5\%), hepatitis $(6.5 \%)$, and rash $(4.8 \%)$. The incidence of severe adverse events (grades 3-5) was $5.6 \%$ of all patients. Six of four hundred fourteen patients (1.4\%) had all grade immune-related pneumonitis, whereas 3 patients had severe pneumonitis (grade 3-5) and were treated with high dose systemic steroids intravenously. One patient with grade 1 pneumonitis did not receive systemic steroids and symptoms resolved after holding the ICI. Another 2 patients with grade 2 pneumonitis were treated with oral prednisolone until recovery. No patient was treated with infliximab. Hepatitis was reported in 27 of 414 patients $(6.5 \%)$, of which 22 of 27 patients had grade 1-2 hepatitis and did not require systemic steroids. Only 5 patients with grade 3-4 hepatitis were treated with systemic steroids. All grade hepatitis was completely resolved in 23 of 27 patients $(85.2 \%)$ at the time of data cut-off.

Median time to onset of overall irAEs was 63 days (range 1-526). Pancreatitis and pneumonitis had the earliest irAE onset of 30 and 34 days, respectively. Hypothyroidism and hypophysitis had the longest median time to resolution of all irAEs (186 and 208 days, respectively). Twenty-two of ninety-eight patients with irAEs had a permanent discontinuation of ICIs due to toxicity. The majority of irAEs in this study completely resolved (85.7\%), either with irAE-specific treatments or discontinuation of immunotherapy. ICIs were rechallenged in 68 of 98 patients with irAE (69.4\%) when the
irAEs recovered to grade 1 or less. Subsequently, 11 of 68 patients with irAEs (11.2\%) had a recurrence of irAEs after ICI rechallenge. Pancreatitis (40\%), skin rash $(23.5 \%)$, and hepatitis $(17.7 \%)$ were the most common manifestations of irAE recurrence. No irAE-related death (grade 5) was observed in this study. The median duration of follow-up was 9.6 months. Patients with irAEs had significant longer median duration of followup when compared with patients without irAEs (13.4 vs 8.1 months; $p=0.005$ ).

Univariate and multivariate analyses for irAE occurrence Odds Ratio (OR) are depicted in Table 4. In the multivariate analysis for occurrence of irAE OR, history of hypothyroidism (OR=7.21, 95\% CI 2.38-21.81; $p<0.001)$, immunotherapy use in clinical trial settings $(\mathrm{OR}=3.43,95 \%$ CI $2.10-5.60 ; p<0.001)$, and combination of immunotherapy/ immunotherapy $(\mathrm{OR}=5.30$, 95\% CI 1.96-14.33; $p<0.001)$ were independent factors predicting occurrence of irAE. In patients with a previous history of hypothyroidism who were on levothyroxine supplementation without recent levothyroxine dose adjustment, 10 of 15 patients (67\%) developed irAEs after initiation of ICI. Five of 10 patients had worsening hypothyroidism. One patient developed hyperthyroidism, for which levothyroxine supplementation was discontinued. Another 3 patients had hepatitis, pneumonitis, and skin rash, respectively, without worsening of their previous hypothyroidism.

\section{Overall survival}

The median OS of the overall cohort was 15.2 months. Overall, patients with irAEs had significantly longer OS when compared to patients without irAEs (18.2 vs 


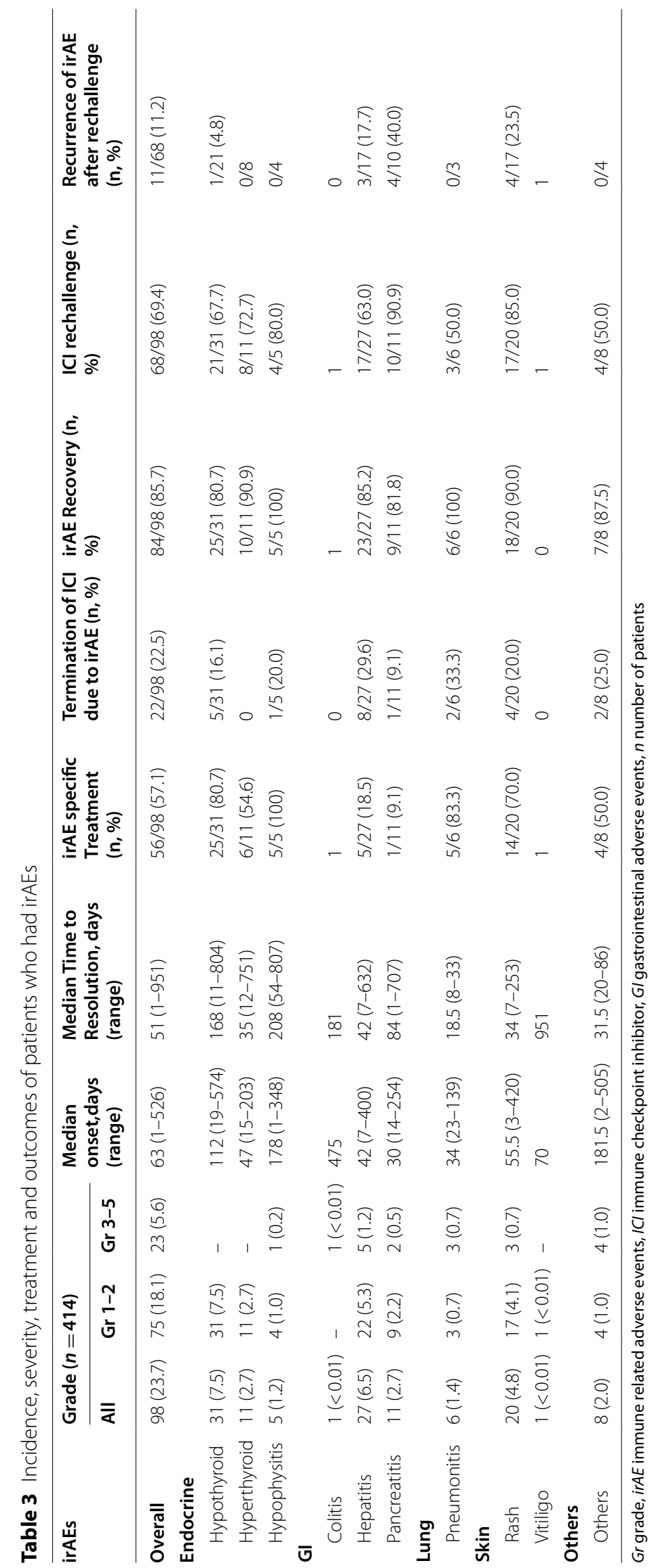


Table 4 Univariate and multivariate logistic regression analyses for Odd Ratio for occurrence of irAE

\begin{tabular}{|c|c|c|c|c|c|c|}
\hline \multirow[t]{2}{*}{ Factors } & \multirow[t]{2}{*}{$\mathrm{N}$} & \multirow[t]{2}{*}{ Event } & \multicolumn{2}{|l|}{ Univariate } & \multicolumn{2}{|l|}{ Multivariate } \\
\hline & & & OR $(95 \% \mathrm{Cl})$ & $p$-value & OR $(95 \% \mathrm{Cl})$ & $p$-value \\
\hline \multicolumn{7}{|l|}{ Age } \\
\hline$<65$ & 226 & 56 & $1.15(0.72,1.81)$ & 0.561 & - & - \\
\hline$\geq 65$ & 188 & 42 & 1 & & & \\
\hline \multicolumn{7}{|l|}{ Sex } \\
\hline Male & 287 & 66 & $0.89(0.55,1.44)$ & 0.627 & - & - \\
\hline Female & 127 & 32 & 1 & & & \\
\hline \multicolumn{7}{|l|}{ Smoking history } \\
\hline Never & 142 & 34 & $1.04(0.60,1.80)$ & 0.889 & - & - \\
\hline Ever & 142 & 33 & 1 & & & \\
\hline Unknown & 130 & 31 & $1.03(0.59,1.81)$ & 0.906 & - & - \\
\hline \multicolumn{7}{|l|}{ History of hypothyroidism } \\
\hline No & 399 & 89 & 1 & & & \\
\hline Yes & 15 & 9 & $5.22(1.81,15.07)$ & 0.002 & $7.21(2.38,21.81)$ & $<0.001$ \\
\hline \multicolumn{7}{|l|}{ Histology Subtype } \\
\hline Squamous & 79 & 21 & $1.21(0.69,2.12)$ & 0.499 & - & - \\
\hline Non-Squamous & 335 & 77 & 1 & & & \\
\hline \multicolumn{7}{|l|}{ Treatment Setting } \\
\hline Clinical trial & 161 & 53 & $3.17(1.99,5.07)$ & $<0.001$ & $3.43(2.10,5.60)$ & $<0.001$ \\
\hline Non-clinical trial & 253 & 39 & 1 & & 1 & \\
\hline \multicolumn{7}{|l|}{ Immunotherapy combination } \\
\hline Monotherapy & 322 & 72 & 1 & & 1 & \\
\hline $\mathrm{ICl}+\mathrm{ICl}$ & 19 & 11 & $4.77(1.85,12.32)$ & 0.001 & $5.30(1.96,14.33)$ & 0.001 \\
\hline $\mathrm{ICl}+$ Chemotherapy/others & 73 & 15 & $0.90(0.34,5.04)$ & 0.736 & $0.82(0.42,1.58)$ & 0.550 \\
\hline
\end{tabular}

$N$ number of patients, $O R$ odd ratio, $I C l$ immune checkpoint inhibitor

13.9 months; $p=0.019$ ) (Fig. 1A). Univariate and multivariate analyses for OS are shown in Table 5, which demonstrate that irAE occurrence was an independent prognostic factor for OS (HR 0.70, 95\% CI 0.51-0.96; $p=0.028)$. In the patients who received ICIs as a first line treatment for metastatic disease, the median OS of patients who had irAEs was 18.2 months, compared with 14.4 months for patients without irAEs (HR 0.63, 95\% CI $0.42-0.96$; $p=0.032$ ) (Fig. 1B). However, there was no statistically significant difference among patients who received ICIs as a second or later line treatment for metastatic disease (20.8 vs 12.6 months; $p=0.300)$, and severity of irAE grade by the CTCAE version $4.0(p=0.064)$ (Figs. 1, and 2C and A). However, solid tumor patients with irAEs who continued ICIs after the first occurrence of irAEs had significantly longer OS when compared to patients who ICIs were discontinued due to irAEs (23.9 vs. 14.7 months; $p=0.013$ ) (Fig. $2 \mathrm{~B}$ ).

\section{Discussion}

We report the largest multicenter study that describes the characteristics and outcomes of solid tumor patients treated with immunotherapy in Thailand. The overall incidence of irAEs in Thailand was consistent with the literature $[3,7-11,17,18]$, where more than $90 \%$ of our patients was treated with anti-PD1/PD-L1 monotherapy. However, the incidence of each specific organ systembased irAEs might be slightly different due to various types of ICIs (or its combinations) used, different dosages, as well as unique patient populations. Several studies suggest that the tumor microenvironment and gut microbiota may also influence the risk and patterns of irAEs $[4,5]$. Since most patients in our study received anti-PD1/PD-L1 monotherapy, irAEs that are most commonly associated with anti-CTLA4 therapy or its combinations thereof, such as colitis, and diarrhea were rare [3]. Endocrinopathies of all grades, especially hypothyroidism, were most commonly reported and the incidence was consistent with previous reports from global and Asian populations [3, 7-11]. The clinical manifestation of thyroid disorders in our patients mostly consisted of early onset thyrotoxicosis or asymptomatic subclinical hypothyroidism, with subsequent transitioning to hypothyroidism that required long-term levothyroxine supplementation. This pattern was consistent with a previous report of immune-related thyroiditis with immune 
A

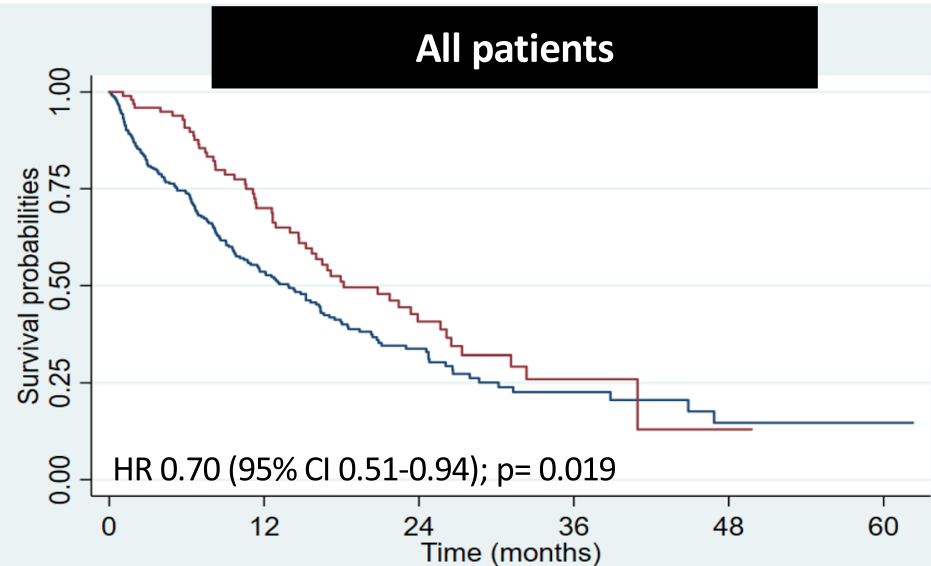

Number at risk

No irAEs 316

irAEs 98

$119 \quad 40$

56

1L Metastatic setting

B

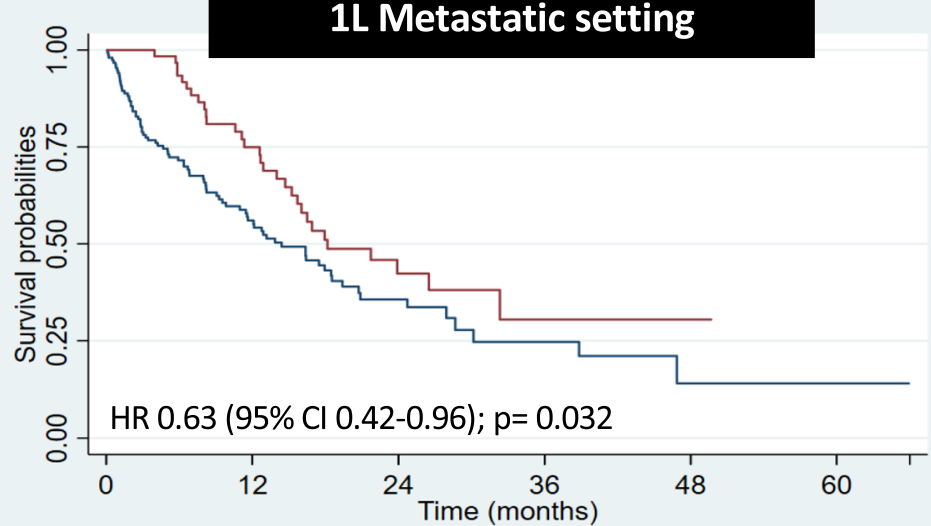

Number at risk

No irAEs 152

irAEs 62

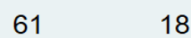

18
12

7

1

37

12

3

1

\section{$\geq 2 \mathrm{~L}$ Metastatic setting}

c

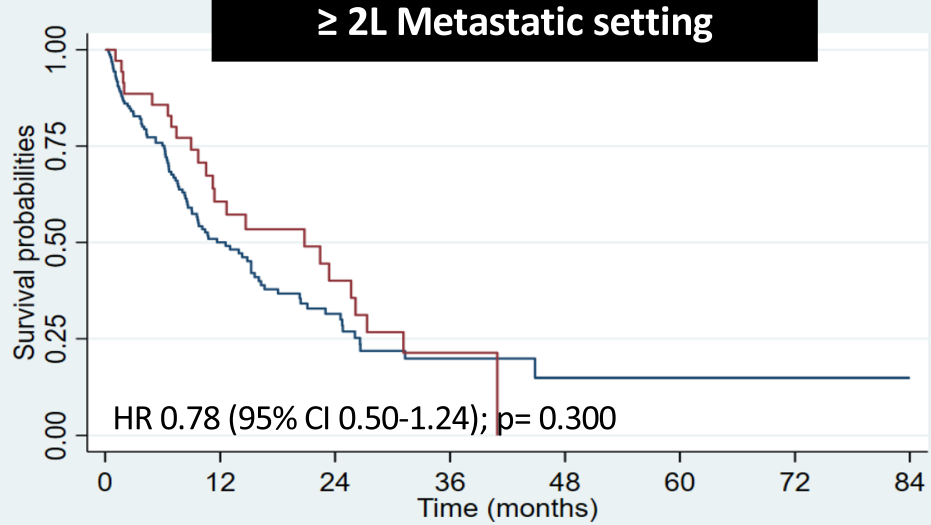

Number at risk

\begin{tabular}{rllllllll} 
No irAEs & 159 & 55 & 21 & 4 & 3 & 3 & 2 & 2 \\
irAEs & 35 & 18 & 9 & 1 & 0 & 0 & 0 & 0 \\
\cline { 3 - 5 } & & & & &
\end{tabular}

Fig. 1 Overall survival (OS) of all solid tumor patients (A), solid tumor patients who received immunotherapy in $1 \mathrm{~L}(\mathbf{B})$, and $\geq 2 \mathrm{~L}(\mathbf{C})$ metastatic settings with and without irAE 
Table 5 Univariate and multivariate Cox regression analysis for overall survival (OS)

\begin{tabular}{|c|c|c|c|c|c|c|}
\hline \multirow[t]{2}{*}{ Factors } & \multirow[t]{2}{*}{$\mathbf{N}$} & \multirow[t]{2}{*}{ OS (months) } & \multicolumn{2}{|l|}{ Univaritate } & \multicolumn{2}{|l|}{ Multivariate } \\
\hline & & & $\mathrm{HR}(95 \% \mathrm{Cl})$ & $p$-value & $\mathrm{HR}(95 \% \mathrm{Cl})$ & $p$-value \\
\hline \multicolumn{7}{|l|}{ Age } \\
\hline$<65$ & 226 & 15.2 & $1.01(0.78,1.30)$ & 0.964 & - & - \\
\hline$\geq 65$ & 188 & 15.7 & 1 & & & \\
\hline \multicolumn{7}{|l|}{ Sex } \\
\hline Male & 287 & 16.0 & $0.93(0.71,1.21)$ & 0.576 & - & - \\
\hline Female & 127 & 15.2 & 1 & & & \\
\hline \multicolumn{7}{|l|}{ Smoking history } \\
\hline Never & 142 & 15.2 & $0.94(0.70,1.27)$ & 0.686 & - & - \\
\hline Ever & 142 & 13.9 & 1 & & & \\
\hline Unknown & 130 & 15.2 & $0.92(0.66,1.28)$ & 0.610 & - & - \\
\hline \multicolumn{7}{|l|}{ Histology Subtype } \\
\hline Squamous & 79 & 15.2 & $1.16(0.86,1.58)$ & 0.335 & - & - \\
\hline Non-Squamous & 335 & 15.2 & 1 & & & \\
\hline \multicolumn{7}{|l|}{ Treatment Setting } \\
\hline Clinical trial & 161 & 16.4 & $0.84(0.65,1.09)$ & 0.192 & $0.90(0.69,1.18)$ & 0.450 \\
\hline Non-clinical trial & 253 & 14.7 & 1 & & 1 & \\
\hline \multicolumn{7}{|l|}{ Immunotherapy combination } \\
\hline Monotherapy & 322 & 15.2 & 1 & & 1 & \\
\hline $\mathrm{ICl}+\mathrm{ICl}$ & 19 & 15.2 & $1.07(0.60,1.92)$ & 0.819 & $1.20(0.67,2.18)$ & 0.539 \\
\hline $\mathrm{ICI}+$ Chemotherapy/other & 73 & 16.0 & $0.89(0.61,1.29)$ & 0.530 & $0.88(0.61,1.28)$ & 0.512 \\
\hline \multicolumn{7}{|l|}{ irAEs } \\
\hline Yes & 98 & 18.2 & $0.70(0.51,0.94)$ & 0.020 & $0.70(0.51,0.96)$ & 0.028 \\
\hline No & 316 & 13.9 & 1 & & 1 & \\
\hline
\end{tabular}

$N$ number of patients, $O S$ overall survival, $I C l$ immune checkpoint inhibitor, irAE immune related adverse events

checkpoint inhibitors $[19,20]$. Similarly, life-threatening irAEs such as immune-related pneumonitis were uncommon with an incidence of $1.4 \%$ in our study, which was comparable with the rate reported in the literature [3, $7-11,17,18,21]$.

In our study, pancreatitis and pneumonitis had the earliest onset of approximately 4 weeks, while hypothyroidism had the latest onset of almost 12 weeks after initiation of ICIs. In a pooled analysis of patients with advanced melanoma who received nivolumab monotherapy, patterns and characteristics of selected treatment-related AEs were described [18]. Skin and GI toxicities had the shortest median time to onset of 5.0 and 7.3 months, respectively [18]. Endocrinopathy occurred at 10.4 weeks, whereas pulmonary toxicity was observed at 8.9 weeks after starting nivolumab [16]. On the other hand, patients treated with ipilimumab had skin, and GI-related AEs after 2-3weeks, and 6 to 7 weeks, respectively [22]. Endocrinopathy occurred after an average of 9weeks of ipilimumab treatment [22]. In our study, skin rash had the shortest median time to resolution of almost 5 weeks, while Weber, et al. reported a median time to resolution of 18 weeks for skin toxicity in advanced melanoma patients treated with nivolumab [18]. In this study, we reported that within the $69 \%$ of patients who were re-challenged with immunotherapy after their irAEs resolved to grade 1 or less, $11 \%$ experienced irAE recurrence. To our knowledge, there are limited data on immunotherapy rechallenge and irAE reoccurrence in the literature. In cases where the ICI continues to benefit patients with non-life-threatening irAEs, ICI rechallenge when appropriate might be possible since the recurrence rate of irAEs was acceptable.

In this retrospective, patients involving in multicenter clinical studies, immunotherapy combination and history of hypothyroidism were independent predictors of irAE occurrence, which is consistent with the literature. Patients in a prospective clinical trial would have more aggressive monitoring of irAE leading to more accurate toxicity reports when compared to those in usual clinical practice or in compassionate usage program. The combination of two ICIs has been known to increase irAE risk when compared to single agents $[17,21]$. Previous retrospective studies reported that elevated levels of baseline thyroid stimulating hormone (TSH), and pre-existing anti-thyroid antibodies were significantly associated with 
A

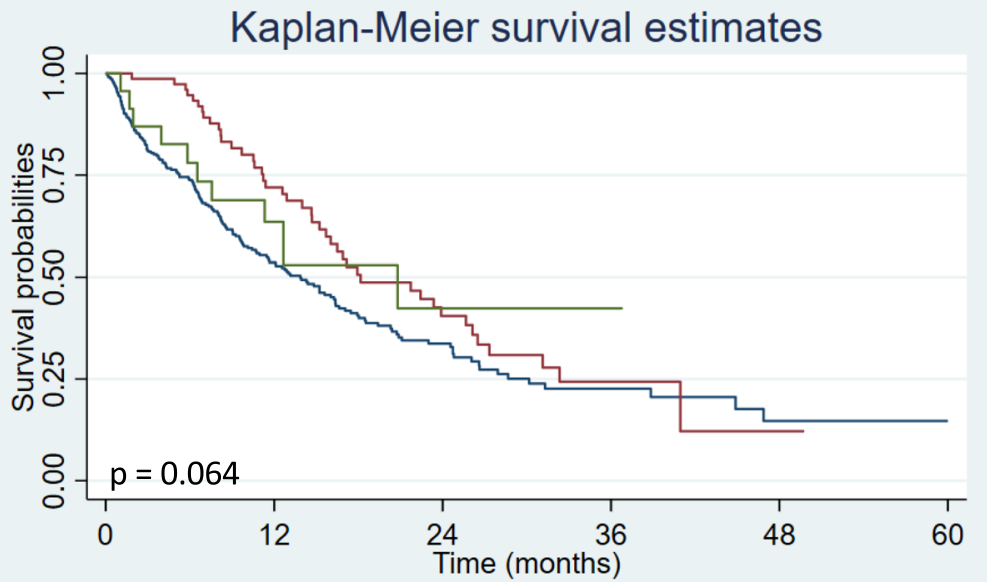

Number at risk

Grade 0316

Grade 1-2 75

Grade 3-5 23

\begin{tabular}{rccccc|}
316 & 119 & 40 & 11 & 4 & 4 \\
75 & 44 & 19 & 3 & 1 & 0 \\
23 & 12 & 2 & 1 & 0 & 0 \\
\hline & Grade 0 & & Grade 1-2 & & Grade 3-5 \\
\cline { 5 - 6 } & & &
\end{tabular}

B

Kaplan-Meier survival estimates

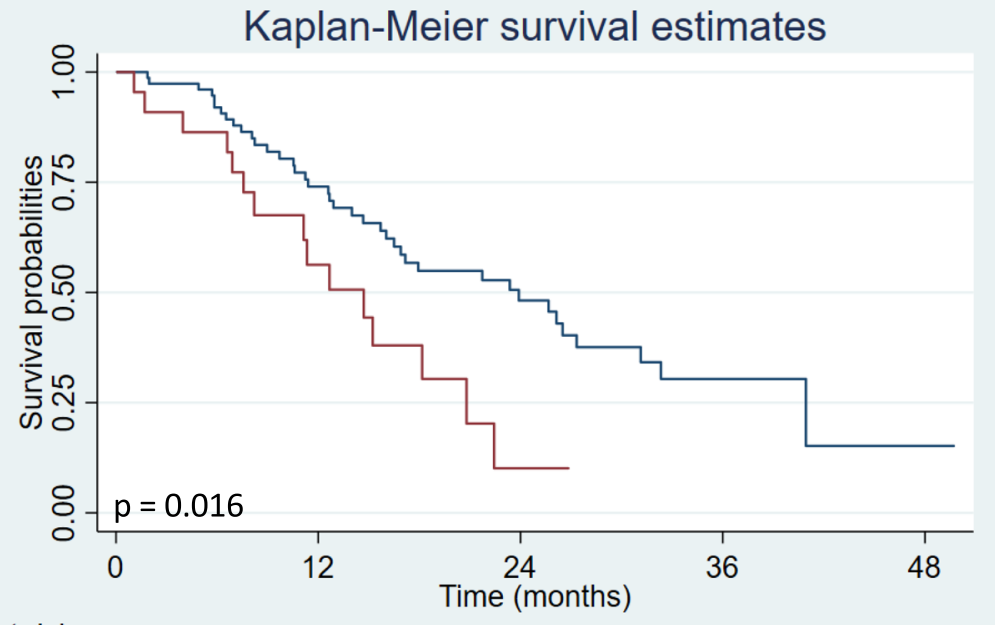

Number at risk

Continue IO 76

Terminate 1022

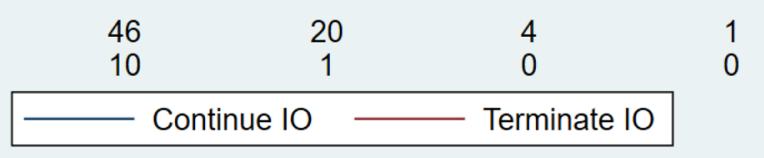

Fig. 2 OS of solid tumor patients by the severity of irAEs using the CTCAE version 4.0 (A), OS of solid tumor patients with irAEs who continued and discontinued ICls after the first irAE occurrence (B)

immune-related thyroid dysfunction in patients treated with ICIs [20, 23].

Our study demonstrated that patients with irAEs had significantly longer OS in both univariate and multivariate analyses. However, patients who developed irAEs had longer follow-up duration when compared with patients who did not. Therefore, survivorship bias should be considered to interpret the relationship between irAEs occurrence and favorable survival. Although the association of irAE occurrence and survival of cancer patients treated with ICIs remains controversial, most studies suggest that irAE occurrence was associated with ICI efficacy [18, 20, 24-27]. A meta-analysis of 30 studies demonstrated a significant association between 
irAE occurrence and better outcome of ICI-treated solid tumor patients, in particular for anti-PD-1 or PD-L1 inhibitors [24].

Our study had several limitations. Though almost 40\% of patients in our study received ICIs in a prospective clinical trial setting, incidences of low grade asymptomatic irAEs might be overlooked and limited in the retrospective part of the study. Survival outcomes might be affected by various tumor types, treatment settings (adjuvant, 1st, and later line metastatic), dosing schedule, and ICI combinations. Moreover, the study might have relatively short follow-up time although almost half of patients in the study received ICIs as the 2nd or later line treatment for metastatic disease. Therefore, survival outcomes in this study should be interpreted with caution.

Since all commercially available ICIs are expensive and not reimbursable by major insurance policies in Thailand, the majority of Thai patients have limited access to these drugs. Though this is the largest multicenter study describing characteristics and outcomes of irAE treatment in Thailand, the study is limited by data collection which occurred in both a prospective (for clinical trials) and retrospective manner. In addition, multiple ICIs and their combinations were included in this study although the majority of patients were treated with antiPD1 or PD-L1 inhibitor monotherapy. The study mostly describes patterns and outcomes of irAEs in solid tumor patients treated with ICIs in Thailand. The association of predictive factors of irAE occurrence and survival should be cautiously interpreted.

\section{Conclusion}

irAEs were commonly observed in Thai cancer patients treated with ICIs. Most irAEs were low-grade and manageable following current practice guidelines. Recurrence of irAEs after ICI rechallenge was not uncommon. Patients who experienced irAEs might have significantly longer OS compared to patients without irAEs. However, OS in this study should be interpreted with caution since it might be affected by various tumor types, treatment settings, dosing schedule, and ICI combinations.

\footnotetext{
Abbreviations

CTLA-4: Cytotoxic T-lymphocyte antigen 4; ECOG: Eastern Cooperative Oncology Group; HCC: Hepatocellular carcinoma; HNSCC: Head and neck squamous cell carcinoma; ICl: Immune checkpoint inhibitors; irAE: Immune-related adverse event; PD-L1: Program death ligand-1; RCC: Renal cell carcinoma; TCC: Transitional cell carcinoma; OR: Odd ratio; OS: Overall survival.

\section{Acknowledgements}

All authors thank Songporn Oranratnachai, MD, and Nintita Sripaiboonkij Thokanit, DrPH for statistical analysis, and Vimvara Vacharathit, PhD for language editing. Nuttapong Ngamphaiboon, MD would like to acknowledge the
}

Research Development Grant from the Ramathibodi Hospital, and Thailand Research Fund (TRF).

\section{Authors' contributions}

Study concepts: NN, SI, VS. Study design: NN, SI, VS. Data acquisition: TS, NS, SI. Quality control of data and algorithms: TS, NN. Data analysis and interpretation: All authors. Statistical analysis: TS, NN. Manuscript preparation: NN. Manuscript editing: All authors. Manuscript review: All authors. All authors have read and approved the manuscript.

\section{Funding}

The study was supported by the Thailand Research Fund (TRF), grant number MRG-6180168 to Nuttapong Ngamphaiboon, MD. TRF provided the funding of the study, and did not involve in study design, results, and manuscript preparation.

\section{Availability of data and materials}

The datasets used and/or analysed during the current study available from the corresponding author on reasonable request.

\section{Declarations}

\section{Ethics approval and consent to participate}

The ethics approval was granted by the ethics committee of Mahidol and Chulalongkorn Universities. Ethics approval was obtained through the ethics committee at the Ramathibodi and Siriraj hospitals, Mahidol university, and Chulalongkorn hospital, Chulalongkorn University. All Ethic Committees approved a waiver of consent for this study as a retrospective chart review. The research involves no more than minimal risk to the subject and is not adversely affect the rights and welfare of the subjects. All patient identifications were protected according to the GCP guideline and not published in the manuscript. All procedures performed in this study were in accordance with the ethical standards of the institutional research committee and with the 1964 Helsinki declaration and its later amendments or comparable ethical standards.

\section{Consent for publication}

Not applicable.

\section{Competing interests}

All authors declare no conflict of interest.

\section{Author details}

${ }^{1}$ Medical Oncology Unit, Department of Medicine, Faculty of Medicine, Ramathibodi Hospital, Mahidol University, Bangkok, Thailand. ${ }^{2}$ Division of Medical Oncology, Department of Medicine, Faculty of Medicine Siriraj Hospital, Mahidol University, Bangkok, Thailand. ${ }^{3}$ Division of Medical Oncology, Department of Internal Medicine, Faculty of Medicine, Chulalongkorn University \& The King Chulalongkorn Memorial Hospital, 1873 Rama IV Rd., Pathumwan, Bangkok 10330, Thailand.

Received: 6 July 2021 Accepted: 9 November 2021

Published online: 25 November 2021

\section{References}

1. Pham T, Roth S, Kong J, Guerra G, Narasimhan V, Pereira L, et al. An update on immunotherapy for solid tumors: a review. Ann Surg Oncol. 2018;25(11):3404-12. https://doi.org/10.1245/s10434-018-6658-4.

2. Postow MA, Sidlow R, Hellmann MD. Immune-related adverse events associated with immune checkpoint blockade. N Engl I Med. 2018;378(2):158-68. https://doi.org/10.1056/NEJMra1703481.

3. Hassel JC, Heinzerling L, Aberle J, Bahr O, Eigentler TK, Grimm MO, et al. Combined immune checkpoint blockade (anti-PD-1/anti-CTLA-4): evaluation and management of adverse drug reactions. Cancer Treat Rev. 2017;57:36-49. https://doi.org/10.1016/j.ctrv.2017.05.003.

4. Dubin K, Callahan MK, Ren B, Khanin R, Viale A, Ling L, et al. Intestinal microbiome analyses identify melanoma patients at risk for 
checkpoint-blockade-induced colitis. Nat Commun. 2016;7:10391. https://doi.org/10.1038/ncomms10391.

5. Pitt JM, Vetizou M, Waldschmitt N, Kroemer G, Chamaillard M, Boneca IG et al. Fine-tuning Cancer immunotherapy: optimizing the gut microbiome. Cancer Res. 2016;76(16):4602-7. https://doi.org/10.1158/0008-5472. Can-16-0448.

6. Wolchok JD, Weber JS, Hamid O, Lebbe C, Maio M, Schadendorf D, et al. I pilimumab efficacy and safety in patients with advanced melanoma: a retrospective analysis of HLA subtype from four trials. Cancer Immun. 2010;10:9.

7. Ngamphaiboon N, Tanaka K, Hong R, Wan Ishak WZ, Yen C, Sriuranpong $V$, et al. Phase III KEYNOTE-048 study of first-line pembrolizumab for recurrent/metastatic head and neck squamous cell carcinoma: Asia vs non-Asia subgroup analysis. In: ESMO ASIA, Singapore; 2019. vol Suppl_9. Ann Oncol.

8. Hida T, Kaji R, Satouchi M, Ikeda N, Horiike A, Nokihara H, et al. Atezolizumab in Japanese patients with previously treated advanced non-smallcell lung Cancer: a subgroup analysis of the phase 3 OAK study. Clin Lung Cancer. 2018;19(4):e405-15. https://doi.org/10.1016/j.cllc.2018.01.004.

9. Tomita Y, Fukasawa S, Shinohara N, Kitamura H, Oya M, Eto M, et al. Nivolumab versus everolimus in advanced renal cell carcinoma: Japanese subgroup 3-year follow-up analysis from the phase III CheckMate 025 study. Jpn J Clin Oncol. 2019;49(6):506-14. https://doi.org/10.1093/jjco/ hyz026.

10. Wu YL, Zhang L, Fan Y, Zhou J, Zhang L, Zhou Q, et al. Randomized clinical trial of pembrolizumab vs chemotherapy for previously untreated Chinese patients with PD-L1-positive locally advanced or metastatic non-small-cell lung cancer: KEYNOTE-042 China study. Int J Cancer. 2020. https://doi.org/10.1002/ijc.33399.

11. MokTSK, Wu YL, Kudaba I, Kowalski DM, Cho BC, Turna HZ, et al. Pembrolizumab versus chemotherapy for previously untreated, PDL1-expressing, locally advanced or metastatic non-small-cell lung cancer (KEYNOTE-042): a randomised, open-label, controlled, phase 3 trial. Lancet. 2019;393(10183):1819-30. https://doi.org/10.1016/S0140-6736(18) 32409-7.

12. Common Terminology Criteria for Adverse Events (CTCAE). (2010). https://evs.nci.nih.gov/ftp1/CTCAE/CTCAE_4.03/CTCAE_4.03_2010-0614_QuickReference_8.5x11.pdf.

13. Brahmer JR, Lacchetti C, Schneider BJ, Atkins MB, Brassil KJ, Caterino JM, et al. Management of Immune-Related Adverse Events in patients treated with immune checkpoint inhibitor therapy: American Society of Clinical Oncology clinical practice guideline. J Clin Oncol. 2018;36(17):1714-68. https://doi.org/10.1200/JCO.2017.77.6385.

14. Haanen J, Carbonnel F, Robert C, Kerr KM, Peters S, Larkin J, et al. Management of toxicities from immunotherapy: ESMO clinical practice guidelines for diagnosis, treatment and follow-up. Ann Oncol. 2018;29(Suppl 4):iv264-6. https://doi.org/10.1093/annonc/mdy162.

15. Puzanov I, Diab A, Abdallah K, Bingham CO 3rd, Brogdon C, Dadu R, et al. Managing toxicities associated with immune checkpoint inhibitors: consensus recommendations from the Society for Immunotherapy of Cancer (SITC) Toxicity Management Working Group. J Immunother Cancer. 2017;5(1):95. https://doi.org/10.1186/s40425-017-0300-z.
16. NCCN (2021) Management of Immunotherapy-Related Toxicities Version 1.2021 - February 1, 2021. https://www.nccn.org/professionals/physi cian_gls/pdf/immunotherapy.pdf.

17. Hellmann MD, Paz-Ares L, Bernabe Caro R, Zurawski B, Kim SW, Carcereny Costa E, et al. Nivolumab plus Ipilimumab in advanced non-small-cell lung Cancer. N Engl J Med. 2019;381(21):2020-31. https://doi.org/10. 1056/NEJMoa1910231.

18. Weber JS, Hodi FS, Wolchok JD, Topalian SL, Schadendorf D, Larkin J, et al. Safety profile of Nivolumab Monotherapy: a pooled analysis of patients with advanced melanoma. J Clin Oncol. 2017;35(7):785-92. https://doi. org/10.1200/JCO.2015.66.1389.

19. Iyer PC, Cabanillas ME, Waguespack SG, Hu MI, Thosani S, Lavis VR, et al. Immune-related thyroiditis with immune checkpoint inhibitors. Thyroid. 2018;28(10):1243-51. https://doi.org/10.1089/thy.2018.0116.

20. Sakakida T, Ishikawa T, Uchino J, Chihara Y, Komori S, Asai J, et al. Clinical features of immune-related thyroid dysfunction and its association with outcomes in patients with advanced malignancies treated by PD-1 blockade. Oncol Lett. 2019;18(2):2140-7. https://doi.org/10.3892/ol.2019. 10466.

21. Larkin J, Chiarion-Sileni V, Gonzalez R, Grob JJ, Cowey CL, Lao CD, et al. Combined Nivolumab and Ipilimumab or Monotherapy in untreated melanoma. N Engl J Med. 2015;373(1):23-34. https://doi.org/10.1056/ NEJMoa1504030.

22. Weber JS, Kahler KC, Hauschild A. Management of immune-related adverse events and kinetics of response with ipilimumab. J Clin Oncol. 2012;30(21):2691-7. https://doi.org/10.1200/JCO.2012.41.6750.

23. Kimbara S, Fujiwara Y, Iwama S, Ohashi K, Kuchiba A, Arima H, et al. Association of pre-existing thyroid autoimmunity with the development of thyroid dysfunction induced by nivolumab. J Clin Oncol. 2018;36(suppl):9091.

24. Haratani K, Hayashi H, Nakagawa K. Association of immune-related adverse events with immune checkpoint inhibitor efficacy: real or imaginary? BMC Med. 2020;18(1):111. https://doi.org/10.1186/ s12916-020-01583-0.

25. Rogado J, Sanchez-Torres JM, Romero-Laorden N, Ballesteros Al, PachecoBarcia V, Ramos-Levi A, et al. Immune-related adverse events predict the therapeutic efficacy of anti-PD-1 antibodies in cancer patients. Eur J Cancer. 2019;109:21-7. https://doi.org/10.1016/j.ejca.2018.10.014.

26. Toi Y, Sugawara S, Kawashima Y, Aiba T, Kawana S, Saito R, et al. Association of Immune-Related Adverse Events with clinical benefit in patients with advanced non-small-cell lung Cancer treated with Nivolumab. Oncologist. 2018;23(11):1358-65. https://doi.org/10.1634/theoncologist. 2017-0384

27. Okada N, Kawazoe H, Takechi K, Matsudate Y, Utsunomiya R, Zamami Y, et al. Association between immune-related adverse events and clinical efficacy in patients with melanoma treated with Nivolumab: a multicenter retrospective study. Clin Ther. 2019;41 (1):59-67. https://doi.org/10. 1016/j.clinthera.2018.11.004.

\section{Publisher's Note}

Springer Nature remains neutral with regard to jurisdictional claims in published maps and institutional affiliations.

Ready to submit your research? Choose BMC and benefit from

- fast, convenient online submission

- thorough peer review by experienced researchers in your field

- rapid publication on acceptance

- support for research data, including large and complex data types

- gold Open Access which fosters wider collaboration and increased citations

- maximum visibility for your research: over $100 \mathrm{M}$ website views per year

At BMC, research is always in progress.

Learn more biomedcentral.com/submissions 